\title{
Treatment of Infections Due to Aspergillus terreus Species Complex
}

\author{
Cornelia Lass-Flörl \\ Division of Hygiene and Medical Microbiology, Medical University of Innsbruck, Schöpfstraße 41, \\ 6020 Innsbruck, Austria; Cornelia.lass-floerl@i-med.ac.at
}

Received: 14 June 2018; Accepted: 6 July 2018; Published: 9 July 2018

Abstract: The Aspergillus terreus species complex is found in a wide variety of habitats, and the spectrum of diseases caused covers allergic bronchopulmonary aspergillosis, Aspergillus bronchitis and/or tracheobronchitis, and invasive and disseminated aspergillosis. Invasive infections are a significant cause of morbidity and mortality mainly in patients with hematological malignancy. The section Terrei covers a total of 16 accepted species of which most are amphotericin B resistant. Triazoles are the preferred agents for treatment and prevention of invasive aspergillosis. Poor prognosis in patients with invasive $A$. terreus infections seems to be independent of anti-Aspergillus azole-based treatment.

Keywords: Aspergillus terreus; amphotericin B; resistance; antifungal treatment

\section{Introduction}

Aspergillosis refers to infection by any of the Aspergillus species; the most common pathogens are representatives of the Aspergillus fumigatus species complex, Aspergillus flavus species complex, Aspergillus terreus species complex, and Aspergillus niger species complex. The A. terreus species complex is found in a wide variety of habitats, soil, compost, or dust, and the spectrum of diseases caused covers allergic bronchopulmonary aspergillosis, Aspergillus bronchitis and/or tracheobronchitis, and invasive and disseminated aspergillosis [1,2]. The section Terrei covers A. terreus sensu stricto (s.s.), A. alabamensis, A. allahabadii, $A$. ambiguus, A. aureoterreus, A. bicephalus, A. carneus, A. citrinoterreus, A. floccosus, A. iranicus, A. hortai, A. microcysticus, A. neoafricanus, A. neoindicus, A. niveus, and A. pseudoterreus, giving a total of 16 accepted species [2]. Infections due to the $A$. terreus species complex occur worldwide. However, there are certain geographic locations with frequent occurrences, such as Innsbruck, Austria and Houston, USA [3]. Within the aspergilli, the A. terreus species complex assumes a specific role, as most representatives are amphotericin B resistant [4]. A. terreus is an emerging opportunistic fungus whose clinical incidence has increased in recent years, with invasive diseases constituting nearly $4 \%$ of all invasive aspergilloses [5]. Of special concern is the high mortality in disseminated diseases caused by this species [6]. Here, we review in vitro susceptibility, experimental and clinical outcome data, and overview recommendations on how to treat infections due to the A. terreus species complex.

\section{The Pathogen and Fungal Epidemiology}

Aspergillus section Terrei is widespread throughout the world and found in arable soil, desserts, and compost heaps [2]. A. terreus usually shows cinnamon-brown colonies when growing on Saubouraud Agar at $37^{\circ} \mathrm{C}$ for $48 \mathrm{~h}$ (see Figure 1a). The production of aleurioconidia in A. terreus, A. carneus, $A$. flavipes, and $A$. niveus seems to be unique among the Aspergillus species; these are morphologically distinct lateral conidia (aleurioconidia) which are attached directly to hyphae (see Figure 1b). Whether these features support dissemination or enhanced virulence in vivo is 
presently unknown. Deak et al. [7] showed that these accessory conidia induce inflammation in a pulmonary mouse model of invasive aspergillosis; amphotericin B resistance was shown not to be associated with the production of aleurioconidia. A. terreus is commonly used in industry to produce organic acids and lovastatin. In a prospective international multicenter surveillance study, including 21 countries and 38 centers, an overall prevalence of 5.2\% (370/7116) of A. terreus infections among mold infections was detected [8]. Cases were reported from Europe $(n=261)$, Middle East $(n=70)$, India $(n=19)$, South America $(n=10)$, and North America $(n=10)$. Spain and Austria were the countries with the highest density of $A$. terreus isolates collected in patients [8].

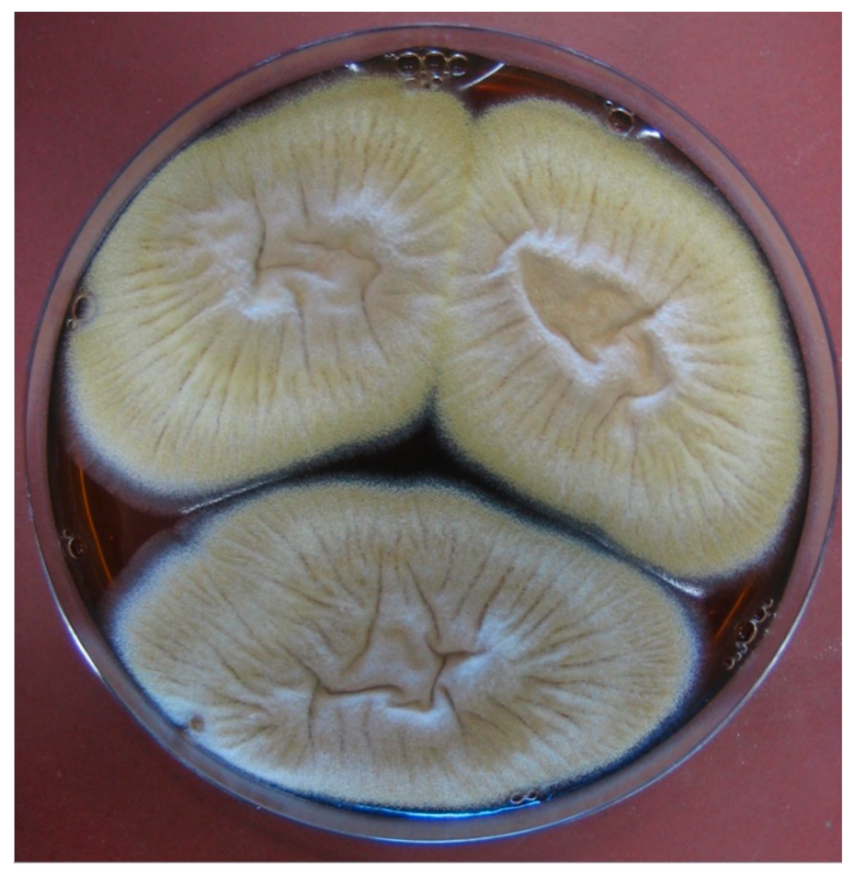

(a)

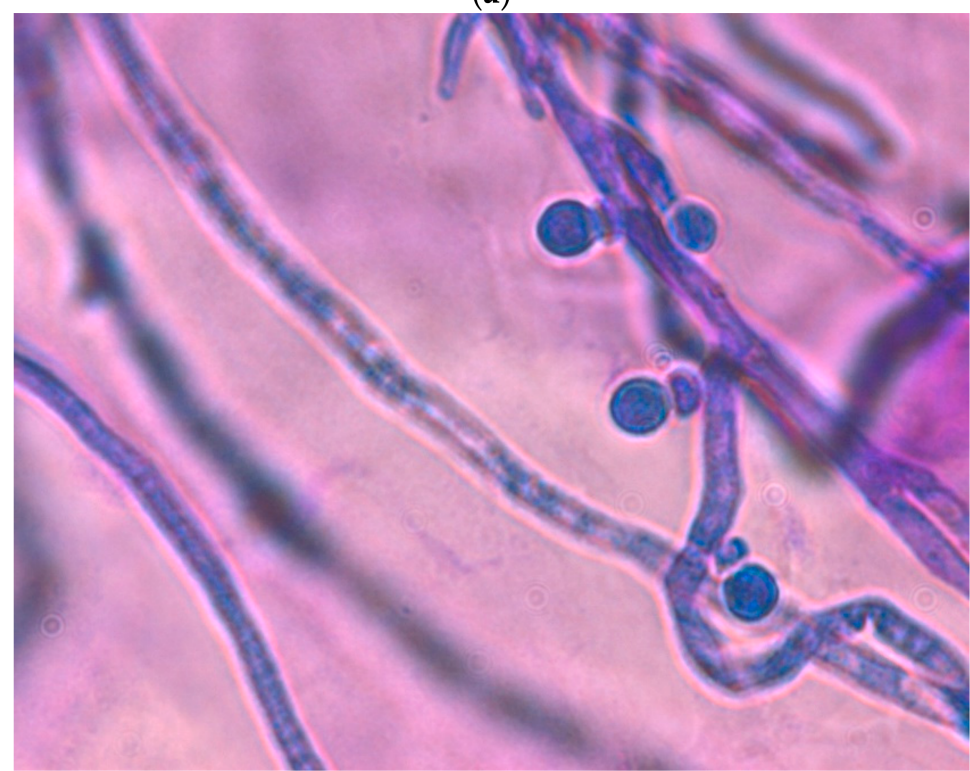

(b)

Figure 1. Cont. 


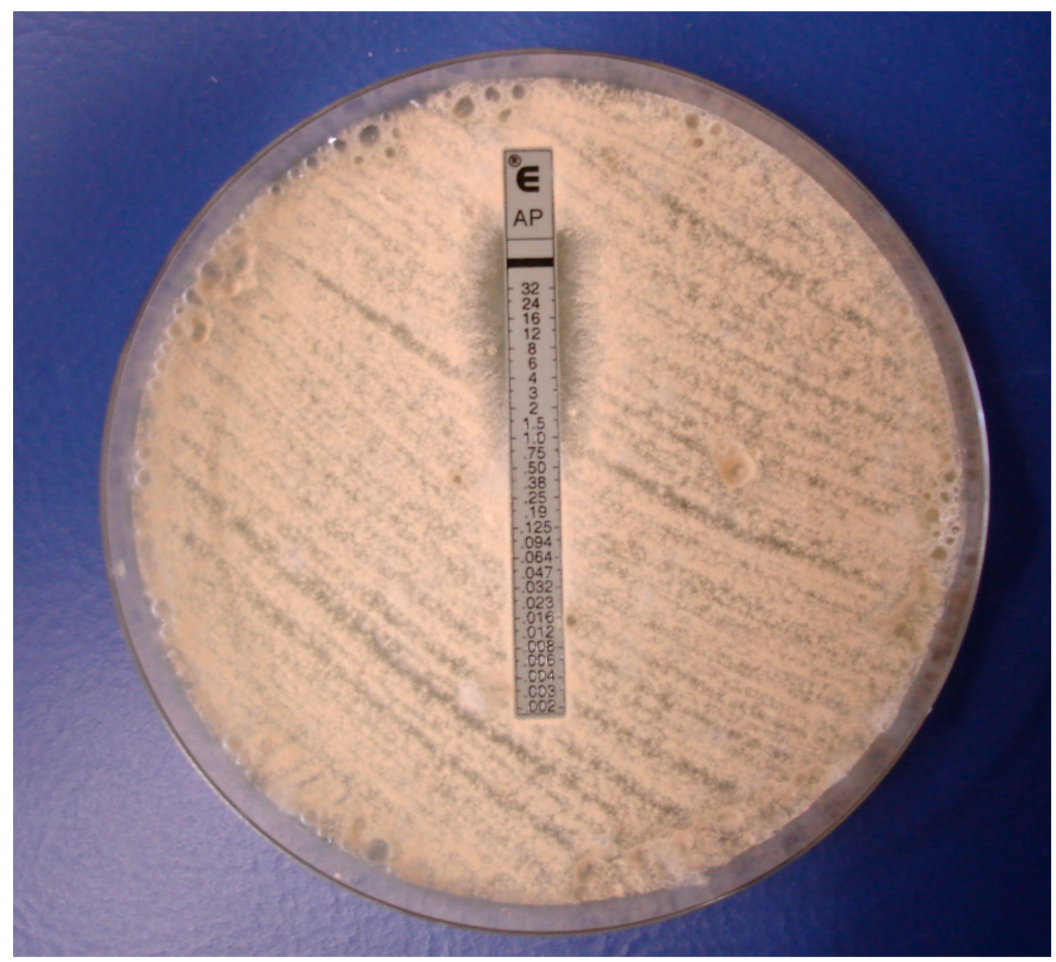

(c)

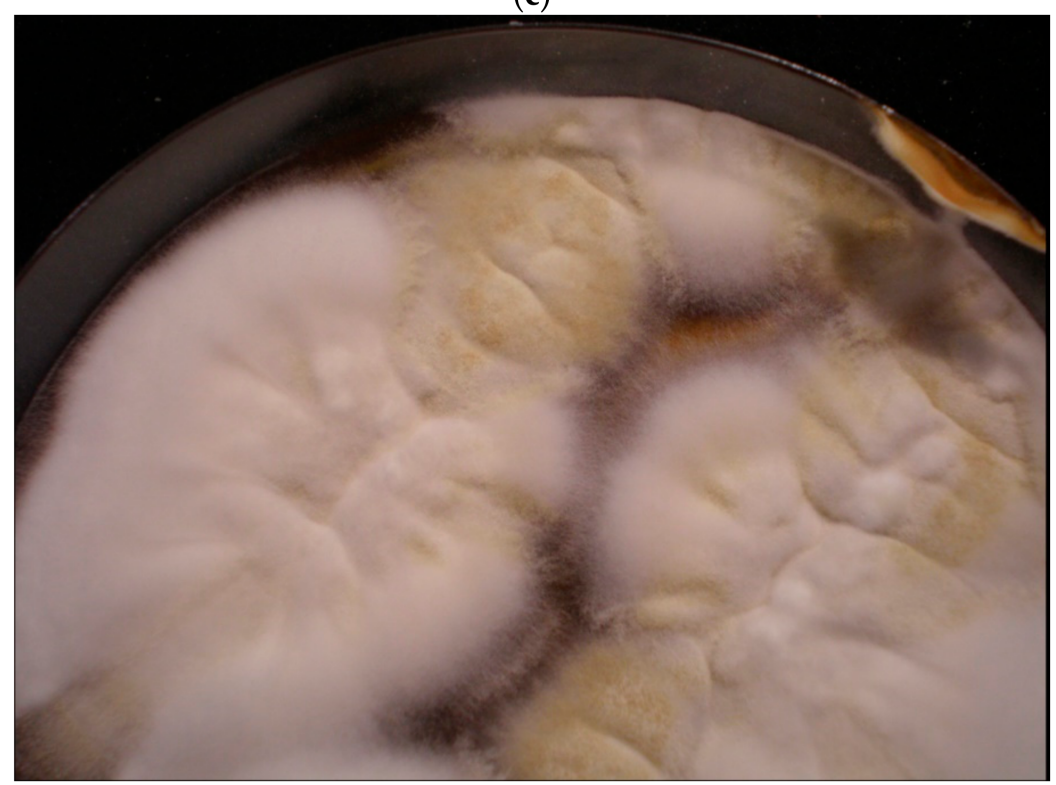

(d)

Figure 1. (a-d) A. terreus sensu strictu displaying typical culture morphology on Sabouraud Agar $\left(48 \mathrm{~h} / 37^{\circ} \mathrm{C}\right)(\mathbf{a})$ and wet mount preparation with lactophenol cotton blue showing aleuroconidia (light microscopy, magnification $\times 1000)(\mathbf{b})$, E-Test ${ }^{\circledR}$ showing amphotericin B resistance $(\mathbf{c})$ and the performance of sectoring by the production of white, fluffy, and conidia-sparse colonies (d).

The reason for this specific epidemiological situation is unclear. A. terreus strains isolated from environmental and clinical sources were genotyped using a novel panel of short tandem repeats [9]. Three major endemic genotypes collected from the Inn region and its side valleys were found to cause the majority of invasive A. terreus diseases in Tyrol, Austria. The genotypes were of the same mating type and persisted for at least 11 years. 


\section{Patients at Risk Suffering from Aspergillus terreus Species Complex}

The at risk population for invasive infections due to Aspergillus section Terrei does not differ from patients suffering from $A$. fumigatus diseases, and comprises mainly individuals with prolonged neutropenia, allogeneic hematopoietic stem cell transplant (HSCT), solid organ transplant (SOT), inherited or acquired immunodeficiencies, and corticosteroid use $[6,10]$. The intensity and duration of neutropenia predict the main risk of infection, including patients with refractory or relapsed acute leukemia treated with reinduction regimens. However, the $A$. terreus species complex also affects non-immunocompromised individuals with prior or current lung disease [8]. Chronic pulmonary aspergillosis is an uncommon and problematic disease thought to affect 240,000 people in Europe. A world-wide, prospective study showed $A$. terreus to be involved in various entities, such as chronic lung diseases $(n=149,39.2 \%)$, hematologic or oncologic malignancies $(n=30,7.4 \%)$, solid organ transplantation $(n=30,7.4 \%)$, diabetes mellitus $(n=24,6.6 \%)$, solid tumors $(n=19,5.1 \%)$, heart $(n=24,6.2 \%)$, renal $(n=14,3.7 \%)$, and liver diseases $(n=4,1.1 \%)$, and others. Underlying diseases comprised invasive aspergillosis $(n=93,25.1 \%)$, allergic broncho-pulmonary aspergillosis $(n=46$, $12.4 \%)$, chronic aspergillosis $(n=42,11.4 \%)$, chronic obstructive pulmonary disease exacerbation ( $n=20,5.5 \%)$, aspergilloma $(n=14,3.7 \%)$, otitis externa $(n=9,2.5 \%)$, wound infections $(n=3,0.7 \%)$, and others [8].

\section{Treatment Recommendations}

The effective management of invasive aspergillosis includes strategies to optimize prevention and early antifungal treatment, immunomodulation, and in some cases, surgery. Overall, treatment strategies should be based on the institutional epidemiology of infections and assessment of individual risk factors.

Triazoles are the preferred agents for treatment and prevention of invasive aspergillosis in most patients [11]. Patients receiving triazole-based therapy for treatment of invasive diseases or prolonged prophylaxis should undergo therapeutic drug monitoring (TDM). This approach supports enhancing therapeutic efficacy of voriconazole and posaconazole. Echinocandins have been shown to be effective only in salvage therapy. Amphotericin B is not recommended; however, in selected cases [12,13], improved treatment outcome was observed under high dose amphotericin B treatment. Combinations of polyenes or azoles with echinocandins suggest additive or synergistic effects in some preclinical studies $[14,15]$. However, variable assay conditions and conflicting results have led to uncertainty as to how to interpret these findings. Early initiation of adequate antifungal therapy is warranted in addition to a diagnostic evaluation [11]. Treatment should be continued for a minimum of 6-12 weeks [11], depending on degree and duration of immunosuppression and the site of infection. Patients with chronic aspergillosis and either pulmonary or general symptoms or progressive loss of lung function should be treated for a minimum of 6 months. Oral itraconazole and voriconazole are the preferred drugs [11]; posaconazole is another option for individuals with adverse events or clinical failure. Hachem et al. investigated 513 patients suffering from hematological malignancies and compared baseline characteristics, antifungal treatment, and outcome between patients infected with $A$. terreus and non-terreus Aspergillus species [10]. A. terreus diseases were associated with a lower response rate to treatment, and a higher rate of mortality when compared to non-terreus infections. In addition, fungal breakthrough infections occurred more often in the A. terreus arm; independent factors associated with final outcome included treatment with azoles $(p<0.0001)$ and Aspergillus species involved $(p=0.043)$.

\section{Amphotericin B}

Amphotericin B, a polyene antifungal drug, was developed in the 1950s and is used intravenously to treat invasive aspergillosis, cryptococcal meningitis, candidiasis, and mucormycosis. In vitro resistance to amphotericin B is rare; few fungal species such as A. terreus, Aspergillus tanneri, Fusarium species, and Scedosporium prolificans show polyene resistance $[16,17]$. The major clinical interest in 
A. terreus infections is related to its amphotericin B resistance (see Figure 1c); retrospective studies revealed high rates (80-90\%) of treatment failure with amphotericin B [18]. The Clinical Laboratory Standard Institute (CLSI) and the European Committee on Antimicrobial Susceptibility Testing (EUCAST) have categorized A. terreus as resistant to amphotericin B [17,19]. Furthermore, 70\% of $A$. terreus isolates investigated showed minimum fungicidal concentrations (MFCs) to be twofold higher than the obtained minimum inhibitory concentrations (MICs). With these findings, the authors underline the poor efficacy against $A$. terreus infections, specifically in neutropenic patients [15]. Animal studies confirmed resistance of $A$. terreus infections even to high doses of amphotericin $\mathrm{B}$ or its liposomal formulation in murine and rabbit models. However, a single experimental study in neutropenic mice suggested $A$. terreus to be susceptible to high doses of liposomal amphotericin $\mathrm{B}$, achieving high concentrations in the lungs $(4-8 \mathrm{mg} / \mathrm{L})$ [13]. A. terreus is intrinsically resistant to amphotericin $B$, and the underlying mechanisms may be associated with the level of catalase production by this species when compared to A. fumigatus [20-22]. Studying amphotericin B resistant (ATR) and amphotericin B susceptible (ATS) clinical A. terreus isolates showed ATR to possess doubled basal superoxide dismutase (SOD) activity when compared to ATS; in addition, ATR presented an enhanced oxidative stress response (OSR), with significantly higher sod2 mRNA levels and increased catalase transcripts during amphotericin B treatment. The inhibition of SOD and catalase (CAT) proteins rendered ATR to ATS in vitro [20-22].

Hypoxia seems to have a major influence on the activity of amphotericin B against $A$. terreus [23]. A significant decrease in the MICs against amphotericin B was detected, mainly due to the loss of mycelium sterillium zones applying the Etest ${ }^{\circledR}$. In addition, the fungicidal activity of amphotericin $B$ against $A$. terreus was significantly decreased under hypoxia, suggesting a shift to fungistatic activity under low-oxygen conditions. Whether these findings have any clinical relevance needs to be investigated in more detail. The alternative in vivo model Galleria mellonella was applied to study the pathogenicity of ATS and ATR, and to evaluate amphotericin B efficacy in vivo [24]. Larvae were susceptible to $A$. terreus infection in an inoculum-size and temperature dependent manner, and in vitro susceptibility to amphotericin B correlated with in vivo outcome, defining an amphotericin $B$ susceptible strain cluster of $A$. terreus [24]. Usually, the MICs of amphotericin B are generally $\geq 2 \mathrm{mg} / \mathrm{L}$ (see Figure 1c), but low MICs (0.06-0.125 mg/L) have also been reported for the $A$. terreus species complex. To our knowledge, the real origin of ATS is unknown; ATS may exist in the environment or may reflect an accidental product of sectoring of ATR on drug-free medium, or may result from both [25]. In a clinical setting, ATS occurs rarely, and we have shown that spontaneous culture degeneration of ATR leads to the emergence of multiple sectors (see Figure 1d), which in turn displayed low MICs against amphotericin B, hence featuring ATS. Susceptibility and virulence studies of sector subcultures (ATSec), ATR, and ATS strains showed ATSec harbouring amphotericin B MICs ranging from 0.12 to $0.5 \mu \mathrm{g} / \mathrm{mL}$, and G. mellonella survival studies revealed an enhanced virulence of ATSec [25]. With regard to antifungal susceptibility testing, this is a very important finding; any colony selected for inoculum preparation should be free of sectoring. Otherwise, MIC data may be unstable in repeating tests, further complicating the interpretation of MIC and in vivo outcome. We noticed that sectoring frequently takes place on various culture media in ATR.

\section{Azoles}

Voriconazole is the drug of choice for first-line treatment of invasive aspergillosis including A. terreus [26]. Limited data are available for itraconazole, posaconazole, and isavuconazole. Four (44\%) out of nine patients were treated with posaconazole (salvage therapy) and responded favorably, whilst the success rate of lipid amphotericin B formulations was only $19 \%$. There is limited clinical experience on the efficacy of the echinocandins in the treatment of $A$. terreus infections. A murine model of A. terreus disseminated infection, with isolate MICs ranging from $0.12 \mathrm{mg} / \mathrm{L}$ to $4 \mathrm{mg} / \mathrm{L}$ in evaluated voriconazole treatment [27]. Improved survival and a reduced fungal load in brain and kidney were given in strains displaying MICs $\leq 1 \mathrm{mg} / \mathrm{L}$. Few experimental data showed that high itraconazole 
doses may be effective in the treatment of animals infected with $A$. terreus. Posaconazole has shown efficacy in treating invasive infections by $A$. terreus in rabbits and mice.

Recently, a considerable amount of azole resistance in Aspergillus section Terri was observed; however, whether the latter finding correlates with in vivo results is presently unknown [28]. The emergence of $A$. terreus sensu lato (s.l.) isolates with reduced azole-susceptibility was reported in patients suffering from cystic fibrosis [29]. In a worldwide study performed, approximately $5 \%$ of all tested A. terreus s.s. isolates were resistant to posaconazole in vitro [28]. In Austria, Germany, and the UK, posaconazole resistance was higher than $10 \%$ in all A. terreus s.s. isolates. The majority of isolates was identified as A. terreus (86.8\%), followed by A. citrinoterreus (8.4\%), A. hortai (2.6\%), A. alabamensis $(1.6 \%)$, A. neoafricanus $(0.2 \%)$, and A. floccosus $(0.2 \%)$. Posaconazole resistance differed geographically and ranged from $0 \%$ in the Czech Republic, Greece, and Turkey, to $13.7 \%$ in Germany. In contrast, azole resistance among cryptic species was rare, and was observed only in one A. citrinoterreus and one A. alabamensis isolate. Resistance against itraconazole and voriconazole was not detected [28]. Azole resistance in $A$. terreus s.s. and $A$. fumigatus is associated with mutations and alterations of the lanosterol-14- $\alpha$ steroldemethylase gene (Cyp51A), a key protein in the ergosterol biosynthesis pathway [30]. However, aside from mutations in the primary target gene, other less known mechanisms (e.g., efflux pumps, overexpression of cyp51) were also found to be involved in azole resistance.

\section{Conclusions}

The effective management of invasive aspergillosis includes strategies to optimize prevention and early antifungal treatment, immunomodulation, and in some cases, surgery. Overall, treatment strategies should be based on the institutional epidemiology of infections and assessment of individual risk factors. Triazoles (Vorriconazole and isavuconazole) are the preferred agents for the treatment and prevention of invasive aspergillosis due to A. terreus in most patients [11]. The underlying resistance mechanisms of $A$. terreus against amphotericin $B$ are only partly understood. The mode of action of amphotericin B seems to be complex and multifaceted (see Figure 2); amphotericin B interacts in various ways with ergosterol, and hence results in fungal cell membrane disruption leading to intracellular leakage and cell death [22]. In addition, amphotericin B may directly act as a pro-oxidant or anti-oxidant, and contribute to an accumulation of free radicals which have multiple deleterious effects. We demonstrated that the co-application of anti- and pro-oxidants significantly affects amphotericin B efficacy in an antithetic manner [21]. Antioxidants and ROS-scavenging agents increased amphotericin B tolerance in susceptible A. terreus strains, while pro-oxidants rendered them resistant to susceptible strains; this in vitro data was confirmed in vivo by applying Galleria mellonella animal models. Whether such management is of clinical interest needs to be studied in detail. 


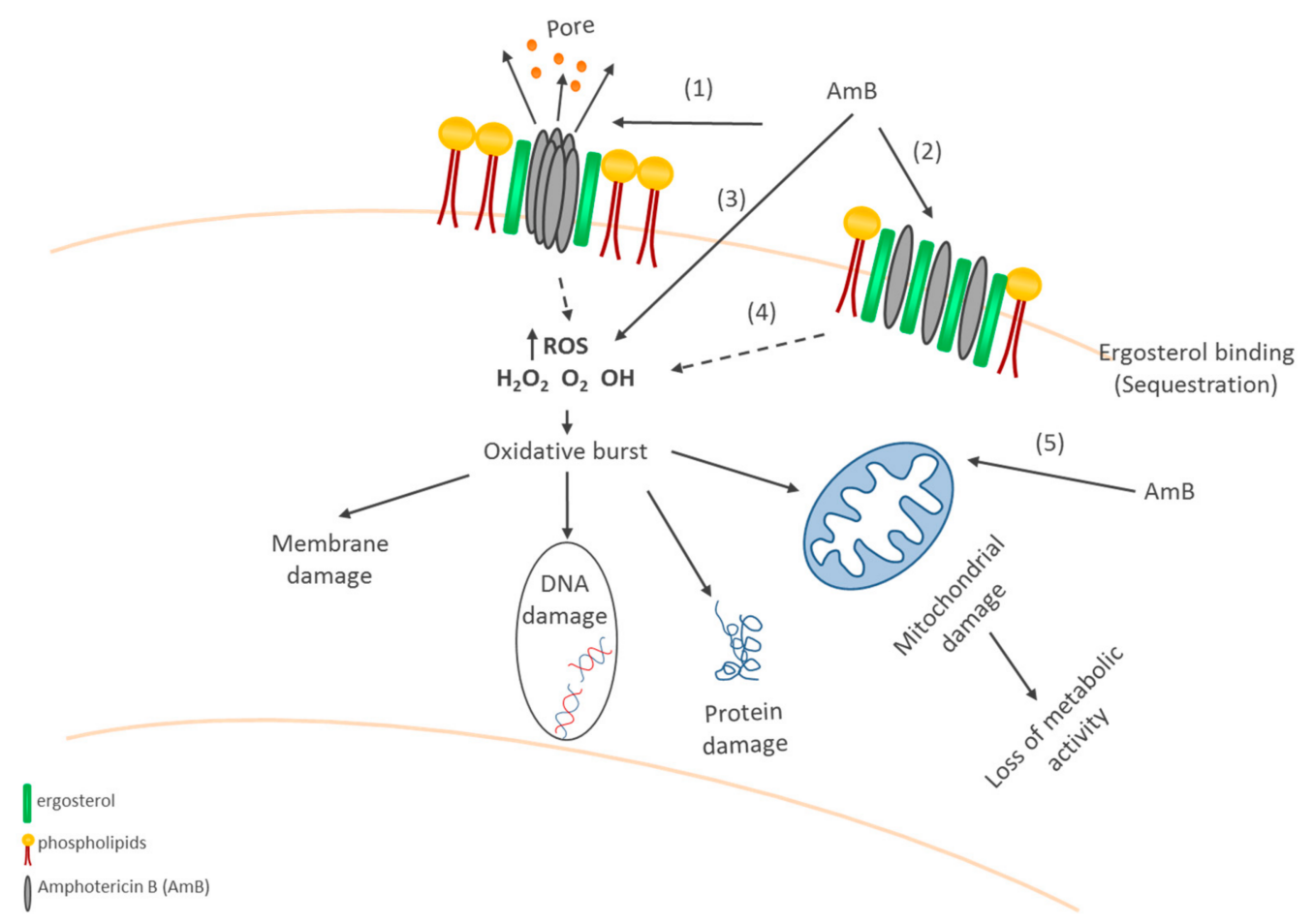

Figure 2. Amphotericin B: Mode of action on A. terreus. There exist two major mechanisms of amphotericin $B(A m B)$ : pore formation in fungal cell membranes $(1,2,3)$ and induction of oxidative stress $(3,4,5)$ causing cell permeability and cell death. Amphotericin B may act as an oxidant as well as antioxidant [22]. Several AmB molecules penetrate the cell, where oxidation takes place, and radicals emerge.

Funding: This research was funded by Christian Doppler Laboratory for Invasive Fungal Infektions.

Conflicts of Interest: The author declares no conflict of interest.

\section{References}

1. Iwen, P.C.; Rupp, M.E.; Langnas, A.N.; Reed, E.C.; Hinrichs, S.H. Invasive pulmonary aspergillosis due to Aspergillus terreus: 12-year experience and review of the literature. Clin. Infect. Dis. 1998, 26, 1092-1097. [CrossRef] [PubMed]

2. Samson, R.A.; Peterson, S.W.; Friswad, J.C.; Varga, J. New species in Aspergillus section Terrei. Stud. Mycol. 2011, 69, 39-55. [CrossRef] [PubMed]

3. Lass-Flörl, C.; Grif, K.; Kontoyiannis, D.P. Molecular typing of Aspergillus terreus isolates collected in Houston, Texas, and Innsbruck, Austria: Evidence of great genetic diversity. J. Clin. Microbiol. 2007, 45, 2686-2690. [CrossRef] [PubMed]

4. Blum, G.; Perkhofer, S.; Haas, H.; Schrettl, M.; Würzner, R.; Dierich, M.P.; Lass-Flörl, C. Potential basis for amphotericin B resistance in Aspergillus terreus. Antimicrob. Agents Chemother. 2008, 52, 1553-1555. [CrossRef] [PubMed]

5. Pastor, F.J.; Guarro, J. Treatment of Aspergillus terreus infections: A clinical problem not yet solved. Int. J. Antimicrob. Agents 2014, 44, 281-289. [CrossRef] [PubMed]

6. Lass-Flörl, C.; Griff, K.; Mayr, A.; Petzer, A.; Gastl, G.; Bonatti, H.; Freund, M.; Kropshofer, G.; Dierich, M.P.; Nachbaur, D. Epidemiology and outcome of infections due to Aspergillus terreus: 10-year single centre experience. Br. J. Haematol. 2005, 131, 201-207. [CrossRef] [PubMed] 
7. Deak, E.; Nelson, M.; Hernández-Rodríguez, Y.; Gade, L.; Baddley, J.; Momany, M.; Steele, C.; Balajee, S.A. Aspergillus terreus accessory conidia are multinucleated, hyperpolarizing structures that display differential dectin staining and can induce heightened inflammatory responses in a pulmonary model of aspergillosis. Virulence 2011, 2, 200-207. [CrossRef] [PubMed]

8. Risslegger, B.; Zoran, T.; Lackner, M.; Aigner, M.; Sánchez-Reus, F.; Rezusta, A.; Chowdhary, A.; Taj-Aldeen, S.J.; Arendrup, M.C.; Oliveri, S.; et al. A prospective international Aspergillus terreus survey: An EFISG, ISHAM and ECMM joint study. Clin. Microbiol. Infect. 2017, 23, 776.e1-776.e5. [CrossRef] [PubMed]

9. Lackner, M.; Coassin, S.; Haun, M.; Binder, U.; Kronenberg, F.; Haas, H.; Jank, M.; Maurer, E.; Meis, J.F.; Hagen, F.; et al. Geographically predominant genotypes of Aspergillus terreus species complex in Austria: A microsatellite typing study. Clin. Microbiol. Infect. 2016, 22, 270-276. [CrossRef] [PubMed]

10. Hachem, R.; Ribeiro Gomes, M.Z.; el Helou, G.; el Zakhem, A.; Kassis, C.; Ramos, E.; Jiang, Y.; Chaftari, A.; Raad, I.I. Invasive aspergillosis caused by Aspergillus terreus: An emerging opportunistic infection with poor outcome independent of azole therapy. J. Antimicrob. Chemother. 2014, 69, 3148-3155. [CrossRef] [PubMed]

11. Ullmann, A.J.; Aguado, J.M.; Arikan-Akdagli, S.; Denning, D.W.; Groll, A.H.; Lagrou, K.; Lass-Flörl, C.; Lewis, R.E.; Munoz, P.; Verweij, P.E.; et al. Diagnosis and management of Aspergillus diseases: Executive summary of the 2017 ESCMID-ECMM-ERS guideline. Clin. Microbiol. Infect. 2018, 24, e1-e38. [CrossRef] [PubMed]

12. Steinbach, W.J.; Marr, K.A.; Anaissie, E.J.; Azie, N.; Quan, S.; Meier-Kriesche, H.; Apewokin, S.; Horn, D.L. Clinical epidemiology of 960 patients with invasive aspergillosis from the PATH Alliance registry. J. Infect. 2012, 65, 453-464. [CrossRef] [PubMed]

13. Lewis, R.E.; Albert, N.P.; Liao, G.; Wan, W.; Prince, R.A.; Kontoyiannis, D.P. High-dose induction liposomal amphotericin B followed by de-escalation is effective in experimental Aspergillus terreus pneumonia. J. Antimicrob. Chemother. 2013, 68, 1148-1151. [CrossRef] [PubMed]

14. Walsh, T.J.; Petraitis, V.; Petraitiene, R.; Field-Ridley, A.; Sutton, D.; Ghannoum, M.; Sein, T.; Schaufele, R.; Peter, J.; Bacher, J.; et al. Experimental pulmonary aspergillosis due to Aspergillus terreus: Pathogenesis and treatment of an emerging fungal pathogen resistant to amphotericin B. J. Infect. Dis. 2003, 188, 305-319. [CrossRef] [PubMed]

15. Graybill, J.R.; Hernandez, S.; Bocanegra, R.; Najvar, L.K. Antifungal therapy of murine Aspergillus terreus infections. Antimicrob. Agents Chemother. 2004, 48, 3715-3719. [CrossRef] [PubMed]

16. Blum, G.; Hörtnagl, C.; Jukic, E.; Erbeznik, T.; Pümpel, T.; Dietrich, H.; Nagl, M.; Speth, C.; Rambach, G.; Lass-Flörl, C. New insights into amphotericin B resistance in Aspergillus terreus. Antimicrob. Agents Chemother. 2013, 57, 1538-1588. [CrossRef] [PubMed]

17. Espinel-Ingroff, A.; Diekema, D.J.; Fothergill, A.; Johnson, E.; Pelaez, T.; Pfaller, M.A.; Rinaldi, M.G.; Canton, E.; Turnidge, J. Wild-type MIC distributions and epidemiological cutoff values for the triazoles and six Aspergillus spp. for the CLSI broth microdilution method (M38-A2 document). J. Clin. Microbiol. 2010, 48, 3251-3257. [CrossRef] [PubMed]

18. Steinbach, W.J.; Benjamin, D.K.; Kontoyiannis, D.P.; Perfect, J.R.; Lutsar, I.; Marr, K.A.; Lionakis, M.S.; Torres, H.A.; Jafri, H.; Walsh, T.J. Infections due to Aspergillus terreus: A multicenter retrospective analysis of 83 cases. Clin. Infect. Dis. 2004, 39, 1922-1928. [CrossRef] [PubMed]

19. European Committee on Antimicrobial Susceptibility Testing. Antifungal Susceptibility Testing (AFST). EUCAST, 2012. Available online: http:/ / www.eucast.org/ (accessed on 27 March 2018).

20. Blatzer, M.; Blum, G.; Jukic, E.; Posch, W.; Gruber, P.; Nagl, M.; Binder, U.; Maurer, E.; Sarg, B.; Lindner, H.; et al. Blocking Hsp70 enhances the efficiency of amphotericin B treatment against resistant Aspergillus terreus strains. Antimicrob. Agents Chemother. 2015, 59, 3778-3788. [CrossRef] [PubMed]

21. Blatzer, M.; Jukic, E.; Posch, W.; Schöpf, B.; Binder, U.; Steger, M.; Blum, G.; Hackl, H.; Gnaiger, E.; Cornelia, L.F.; et al. Amphotericin B resistance in Aspergillus terreus is overpowered by coapplication of pro-oxidants. Antioxid. Redox Signal. 2015, 23, 1424-1438. [CrossRef] [PubMed]

22. Posch, W.; Blatzer, M.; Wilflingseder, D.; Lass-Flörl, C. Aspergillus terreus: Novel lessons learned on amphotericin B resistance. Med. Mycol. 2018, 56, 73-82. [CrossRef] [PubMed]

23. Binder, U.; Maurer, E.; Lackner, M.; Lass-Flörl, C. Effect of reduced oxygen on the antifungal susceptibility of clinically relevant aspergilli. Antimicrob. Agents Chemother. 2015, 59, 1806-1810. [CrossRef] [PubMed] 
24. Maurer, E.; Browne, N.; Surlis, C.; Jukic, E.; Moser, P.; Kavanagh, K.; Lass-Flörl, C.; Binder, U. Galleria mellonella as a host model to study Aspergillus terreus virulence and amphotericin B resistance. Virulence 2015, 6, 591-598. [CrossRef] [PubMed]

25. Jukic, E.; Blatzer, M.; Binder, U.; Mayr, L.; Lass-Flörl, C.; Lackner, M. Impact of morphological sectors on antifungal susceptibility testing and virulence studies. Antimicrob. Agents Chemother. 2017, 61, e00755-17. [CrossRef] [PubMed]

26. Trifilio, S.; Ortiz, R.; Pennick, G.; Verma, A.; Pi, J.; Stosor, V.; Zembower, T.; Mehta, J. Voriconazole therapeutic drug monitoring in allogeneic hematopoietic stem cell transplant recipients. Bone Marrow Transplant. 2005, 35, 509-513. [CrossRef] [PubMed]

27. Salas, V.; Pastor, F.J.; Sutton, D.A.; Calvo, E.; Mayayo, E.; Fothergill, A.W.; Rinaldi, M.G.; Guarro, J. MIC values of voriconazole are predictive of treatment results in murine infections by Aspergillus terreus species complex. Antimicrob. Agents Chemother. 2013, 57, 1532-1534. [CrossRef] [PubMed]

28. Risslegger, B.; Zoran, T.; Lackner, M.; Aigner, M.; Sánchez-Reus, F.; Rezusta, A.; Chowdhary, A.; Taj-Aldeen, S.J.; Arendrup, M.C.; Oliveri, S.; et al. Azole-resistance in Aspergillus terreus and related species: An emerging problem or a rare phenomenon? Front. Micorbiol. 2018, 9, 516.

29. Arendrup, M.C.; Jensen, R.H.; Grif, K.; Skov, M.; Pressler, T.; Johansen, H.K.; Lass-Flörl, C. In vivo emergence of Aspergillus terreus with reduced azole susceptibility and a Cyp51a M217l alteration. J. Infect. Dis. 2012, 206, 981-985. [CrossRef] [PubMed]

30. Chowdhary, A.; Sharma, C.; Meis, J.F. Azole-resistant aspergillosis: Epidemiology, molecular mechanisms, and treatment. J. Infect. Dis. 2017, 216, S436-S444. [CrossRef] [PubMed]

(C) 2018 by the author. Licensee MDPI, Basel, Switzerland. This article is an open access article distributed under the terms and conditions of the Creative Commons Attribution (CC BY) license (http:/ / creativecommons.org/licenses/by/4.0/). 